\title{
Réveil, réincarnation et restitution : Yves Bonnefoy dans l'Imaginaire musical de Jeremy Thurlow
}

\author{
Carole BOURNE-TAYLOR (Oxford) ${ }^{1}$
}

\section{Summary}

In his sensitive and original compositions inspired by poems by Yves Bonnefoy, Jeremy Thurlow seems to have intuitively rather than consciously captured the poet's own longstanding preoccupations, especially his never-ending quest for Presence, which is the cornerstone of his poetic endeavour. In its duplicity, image is a threat to Presence, yet they are inextricably linked. A phenomenologically-inspired analysis, based upon Vladimir Jankélévitch's insights, reveals that music allows a way out of the closed structures of image and concept. Thurlow's musical rendering of Bonnefoy's poetic intuitions enact the true vocation of poetry by allowing the sounds to release their full revelatory potential.

L'œuvre d'Yves Bonnefoy est immense, mouvante, indivise, qui englobe la poésie, la poétique (au sens aussi de poéthique ; cf. Pinson 1995, 161s), la critique et la traduction. En son cœur, irradiante, la poésie fait signe et sens, délinéant une inlassable et exigeante quête de la Présence, phénomène ambigu s'il en est. Souvenance de cette Présence, la poésie est vouée à la restitution d'un « rapport intensifié au monde » (Bonnefoy 2010a, 421). En effet, la conception bonnefoyenne de la poésie repose sur «l'intensification du langage : plus de présence, plus de plénitude immédiate » (Bonnefoy 2010a, 421).

C'est bien dans le sens d'une telle intensification qu'il convient d'appréhender la stéréophonie d'un espace-temps musical qui permet à l'œuvre poétique de réaliser sa vraie vocation, toute aporétique soit-elle : celle de dégager la pensée de son fatras conceptuel et utilitaire et de la recharger d'une force émotionnelle et existentielle. En outre, comme l'a formulé Vladimir Jankélévitch, la musique accomplit le " miracle » de la " présence immédiate " qui paradoxalement surgit d'un "horizon lointain" (Jankélévitch 1983b, 64).

C'est bien dans cette mouvance que se situe le compositeur britannique Jeremy Thurlow qui prolonge dans son œuvre musicale les intuitions de l'œuvre poétique de Bonnefoy, d'abord hasard de lecture avant de se cristalliser en source d'inspiration. ${ }^{2}$ L'écoute des compositions permet au lecteur-auditeur de ré-interroger le travail de l'interprétation, qui 
consiste à repousser les frontières, à libérer l'esprit d'une œuvre en son mystère et à distiller ce mystère.

C'est en termes de filiations et de restitution que nous pouvons aborder l'Imaginaire musical $^{3}$ de Jeremy Thurlow, lui-même spécialiste de Dutilleux lequel est uni à Bonnefoy par une fascination pour Baudelaire (cf. Froidefond 2014, 117-133). D'emblée se met en place une concentricité compliquée. Le paradigme idéal est celui de la fin du XIX siècle tel qu'il est représenté par Baudelaire et Debussy. Aussi n'est-il pas surprenant que Thurlow ait recours au terme de 'correspondances > pour décrire le lien entre ses adaptations et leurs sources d'inspiration. Il prend soin de préciser que ces compositions inspirées de Bonnefoy ( sonnent français `. Le riche motif de la filiation compose un entrelacement pour le moins complexe au sein d'un vaste corpus qui s'enrichit d'adaptations de Keats, d'Emily Dickinson, de Virginia Woolf et de Stephen Romer, tous unis par une forte tonalité élégiaque, par une inlassable interrogation sur la nature insaisissable de leur objet, auteurs chez qui vivre, écrire et mourir configurent une présence pleine. Thurlow resserre les liens d'une filiation déjà établie, qui peut ainsi s'accomplir en tant que communauté esthétique d'esprits hantés par la perte, souvent enclins au ressassement, où point cependant l'espoir, grande notion bonnefoyenne s'il en est.

Le paradigme musical informe l'œuvre du grand poète français que Patrick Quillier qua-

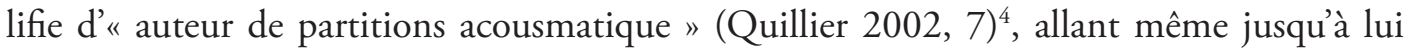
conférer le titre de " maître de chapelle ». On songe au "Coup de dés " de Mallarmé, mais aussi à Virginia Woolf qui disait concevoir ses ouvrages comme des partitions de musique. ${ }^{5}$

Poète du signifié plus que du signifiant, Bonnefoy joue en fait sur les deux versants : plutôt que de succomber à l'enivrement logolâtre, la signifiance est avènement vibratoire de la présence, aspiration existentielle plus que textuelle. De son propre aveu, il s'attache à " réaccorder» (Bonnefoy 1990, 20) la langue afin que « les relations de sonorité, de rythmes, rapprochent les mots d'une façon qui préserve dans notre écoute leur qualité matérielle » (Bonnefoy 1990, 263). La justesse de ton mobilise toutes les ressources sonores du poème :

Quand on écoute le son du mot, ce son qui est l'autre moitié mais toujours étouffée du signe, quand on lui donne le droit de laisser vivre ses rythmes, vibrer ses assonances, se déclarer et s'approfondir sa musique, voici qu'on se trouve engagé dans une écriture où, du fait de cet autre rapport des mots entre eux, leur articulation conceptuelle est affaiblie, avec l'idée qu'elle imposait de la réalité et de l'existence. Ce voile s'amincit, parfois se déchire, la présence à l'Un du monde y transparaît, comme un bien, c'est ce que je nomme la poésie. Cette poésie ne dit rien à proprement parler : elle montre. (Bonnefoy 1994, 2)

La substance sonore se dégage de la gangue des mots, s'épanouit en présence, cette présence qui vit sous l'épée de Damoclès du concept. Du registre poético-langagier au registre musicotechnique, une même discontinuité se fait entendre, à même de "retrouver l'amande du poétique» (Bonnefoy 2010a, 315). 
Michèle Finck, éminente spécialiste des rapports entre la poésie et la musique envisagés sous l'angle d'une poétique de la résonance et du rythme, propose de pratiquer une " audiocritique " (Finck 2004, 22) de l'œuvre bonnefoyenne. La texture sonore, acoustique, rythmique constitue un ensemble au sein duquel signe et sens sont consubstantiels. Une telle entreprise consiste à apprécier cette " géologie sonore de la langue " (Finck 2014, 319), "sa matière sonore et rythmique profonde " (Finck 2014, 331), particulièrement en sa qualité de raucité. Si le poète est Orphée et son instrument, la lyre, alors il convient de préciser qu' " une lyre qui ne serait pas ` rauque > risquerait d'être mensongère ». (Finck 2014, 292) La raucité devient ainsi la " clé de la poétique de Bonnefoy " (Finck 2017, 334), une véritable épiphanie musicale : mimant la réalité dans sa modalité de matérialité, de rugosité et de mortalité, elle relève d'un régime bien différent de l'harmonie qui ne serait en fin de compte qu'un leurre, comme les belles images. La poétique bonnefoyenne consiste à réactiver la matière brute d'une signifiance en constante résonance, comme la main qui remue l'eau dans le poème "Deux couleurs ", geste qui conduira, en sa phase ultime, à sa modalité vocale, dissonante, qui est autant émotionnelle que commotionnelle : le cri, qui est aussi un cri d'alarme.

La poésie joue un rôle incontestable dans l'imaginaire musical de Jeremy Thurlow dont la sensibilité littéraire se greffe sur une affinité avec l'esthétique symboliste de Debussy dont il émule à merveille la musique atmosphérique, musique qui oscille entre la présence et l'absence, le proche et le lointain.

C'est la lecture, en 2012, de la traduction d'un recueil de Bonnefoy, en particulier « Deux couleurs ", section du Dans le leurre du seuil, qui incita Thurlow à composer un morceau d'une longueur de 17 minutes pour flûte et piano, qui reprend le premier vers du poème, "Plus avant que l'étoile", en guise de titre.

" Deux couleurs » se compose de deux parties séparées par une ligne en pointillé. Thurlow se rendit vite compte que pris dans sa globalité, le poème avait un trop grand pouvoir de suggestion $^{6}$, d'où le retranchement de la deuxième partie et, par extension, le changement de titre puisque les "deux couleurs » éponymes n'émergent que dans la deuxième partie du poème dans sa relation à la première partie.

En ce qui concerne l'exécution, Thurlow suggère que les vers de Bonnefoy soient lus avant sa composition plutôt qu'ils ne soient imprimés dans le programme. Il importait au compositeur de jouer sur le paradoxe de l'invisibilité d'un poème qui néanmoins continue de conférer une forme imperceptible, inouïe - serions-nous tentés de dire - à la musique. Paradoxe qui veut qu'en s'émancipant du modèle Thurlow y soit doublement fidèle : en effet une telle transposition se situe dans le sillage de la pratique traductionnelle qui faisait partie intégrante de l'entreprise poétique bonnefoyenne. Thurlow, qui parle de son adaptation comme d'un ‘ filtre `, attend de son medium qu'il fasse ressortir certains aspects du texte tout en restant au plus près de sa complexité implicite. ${ }^{7} C^{\prime}$ est l'ambiguïté du poème qui a présidé à la composition d'un morceau de musique instrumentale : imbriqués dans la partition, donc accessibles aux musiciens, les mots sont en revanche dérobés à l'auditoire. Au mieux, la musique peut-elle évoquer ces mots. ${ }^{8}$ On se souvient d'une même réticence chez Dutilleux auquel Thurlow a consacré un ouvrage - qui souhaitait que l'auditeur oublie les références 
et s'abstienne de lire les notices des programmes : choix qui s'est imposé tout naturellement à l'esprit de Thurlow.

Ce qui nous intéresse au premier chef, c'est le pouvoir de la musique - en l'occurrence des œuvres de Thurlow - de cristalliser les linéaments ainsi que les éléments constitutifs de la pensée de Bonnefoy, en particulier l'idée-clé selon laquelle le poème n'est pas la poésie : c'est cette déficience qui constitue le point de départ de notre intérêt pour la mise en musique ou la mise en chant de ces textes. Objet incomplet, le poème sécrète l'image, toujours défaillante, toujours duplice, partagée qu'elle est entre la réalité et le langage, tandis que la poésie serait un champ illimité : " [l]'inachèvement, à jamais, c'est ce qui caractérise la poésie " (Bonnefoy 2010b). La citation nous semble formuler l'enjeu majeur qui justifie la notion de restitution introduite d'emblée ; nous en faisons le fil rouge de notre réflexion.

Ainsi, le poème, d'essence tensionnelle, aspire à la poésie mais il demeure l'instrument d'une quête inlassable. La poésie est bien de l'ordre de ce qui n'en finit pas, ${ }^{9}$ de ce qui n'en finit pas de dire ce qui ne peut être dit. L'idée que Bonnefoy se fait de la poésie comme étant l'infini, comme élan, permet de mesurer la valeur de la mise en musique, qui n'est pas pure stylisation. En effet, ces œuvres peuvent ainsi se hisser au-delà d'elles-mêmes, réaliser leur véritable destinée qui est de combattre l'inclination conceptuelle de la pensée en faveur d'une puissance autre, matérielle. D'où l'importance de la voix dans l'écriture poétique de Bonnefoy, cette voix qui est concrétude acoustique, trace de la présence, donc seule échappatoire de la prison de l'abstraction.

La musique vocale ou instrumentale est l'intrusion salutaire du melos dans le logos, un principe actif, dynamique, catalyseur ; qui décuple et diffuse la puissance du logos, procède à son éclatement. Mobilisé par le potentiel incarnateur du melos qui fait jouer toute la gamme de l'existence de la mélancolie et de la déploration à l'espoir et l'espérance, le logos s'avoue ainsi désabusé, déjoué. D’ailleurs, la musique contemporaine joue beaucoup sur les extrémités de la gamme musicale et les dissonances. ${ }^{10}$ La musique nous transporte dans ce règne qui échappe au leurre ; elle a cette valeur instauratrice que Bonnefoy attribuait à la poésie, ${ }^{11}$ mémoire de la présence, intensification ainsi que questionnement du rapport à la réalité.

Ce parti pris de la part de Thurlow en faveur de la musique instrumentale fut dicté par la nécessité d'échapper à toute détermination, à toute interprétation trop littérale ou prosaïque, et de favoriser l'accès au matériau sonore par une désolidarisation par rapport à l'objet textuel dont l'intégrité est cependant préservée par les paramètres de l'analyse musicale. Ainsi l'écriture poétique bonnefoyenne est libre d'accomplir sa vocation qui est précisément de se libérer des catégories et représentations linguistiques. On aboutit alors à la désécriture que Bonnefoy appelait de ses vœux dans Le nuage rouge (cf. Bonnefoy 1992, 76), ouvrage dans lequel l'incarnation, toujours salutaire, est de l'ordre d'un cri (Bonnefoy 1992, 344). Ainsi, dans la dernière strophe de la première section du poème "Deux couleurs ", un cri se fait entendre, qui, dans sa soudaineté, est doté du pouvoir de "défaire l'étreinte " (Bonnefoy 1975, 273). Ce cri est de l'ordre d'un surgissement, nous laissant entrevoir " cette immédiateté dans le mot, ce plein en lui retrouvé du rapport au monde " (Bonnefoy 1996, 317). Il semble que la poésie s'adonne au miracle musical, voire le produise en se laissant glisser 
au gré de ses signifiants, en une sorte d'apothéose incarnatrice du sens : « [...] du fait des accents dans les mots, ou des assonances qui sont entre eux, ces sons se proposent comme des ébauches de rythmes, dont naît une musique par laquelle à nouveau le corps s'exprime, et cette fois imprégné d'esprit. » (Bonnefoy 1996, 317-318)

La composition musicale poursuit les ambitions exploratoires du travail poétique ; s'élevant contre la clôture de l'image, elle est ouverture, rupture de la bulle verbale, de la beauté formelle de la langue, désormais rendue à l'informe, au mystère, à l'illimité. Il n'est pas surprenant que la boue figure dans ce poème, dans les deuxième et dernière strophes, comme tant d'autres images géologiques dans l'œuvre de Bonnefoy, où se déploie une matérialisation polymorphe et un imaginaire de la pierre.

La musique nous arrache au ‘ monde-image > et à < l'exil conceptuel > (Starobinsky 1982, 27), poursuivant le processus de trans-figuration libératoire amorcé par le langage poétique dont la richesse acousmatique peut désormais retentir au sens où l'entendait Bachelard (Lassus 2010).

Examinons la partition : l'alternance au niveau du tempo gouverne les huit mouvements qui épousent les huit strophes du poème : piacevole, poco mosso pour I et IV, andante pour les mouvements II et $\mathrm{V}$, con moto pour les mouvements III et VII. Les mouvements IV et V sont, au niveau du tempo, le reflet des mouvements I et II. Ces jeux de miroir dans la partition sont cependant perturbés par le fameux misterioso où la flûte joue solo. Il s'agit d'un mouvement beaucoup plus sobre en ce qui concerne les altérations et rythmes, soit pour un effet de pont ou pause, soit pour exprimer un calme pour les paroles qui jouent sur le reflet et l'étoile et le reflet d'étoile. Peut-être n'est-il pas fortuit que ce sixième mouvement mélodieux dénote du reste de la composition : c'est la voix de l'eau, matricielle, originelle, symbole du mystère de la vie et de la mort, symbole de l'insaisissable, qui assume l'énonciation : "Bois, je suis l'eau, brûlée " ("Deux couleurs », v. 41). Il n'est pas surprenant que ce soit la flûte, instrument à vent, donc prolongement du souffle, qui prenne en charge la prosopopée. Thurlow dit avoir cherché à reproduire ce qu'il percevait comme un dialogue sensuel entre le poète et l'eau. À rebours de toute représentation, la présence relève du mystère, ce qui transparait dans la dimension magique, debussyenne de la composition. L'eau, source de vie et annonciatrice de la mort, énonce le mystère de notre condition aussi bien que de la présence, mystère qui prend sa pleine dimension dans le jeu de la fûte. Notons que la fûte entame et termine le morceau sur la note mi, le mi étant la note du reflet et de l'eau dans le misterioso. ${ }^{12}$ D'ailleurs, dans les mouvements II et III la flûte s'élève cantabile au-dessus de la confusion. Dans le mouvement III (à la mesure 89), même quand le piano joue forte (f), la flûte est en ' ff , fortissimo. Après un troisième mouvement, chargé pianistiquement, très dense, cependant très court à cette vitesse, on retrouve un calme, voire un vide en ce qui concerne le piano et un retour de la flûte, intense, qui jouera seule sa mélodie liée pendant plusieurs phrases du mouvement; voix qui exacerbe la puissance expressive et sensorielle du poème. Dans le mouvement IV, la partie flûte ne semble pas liée au mouvement II, elle chante librement au-dessus des rythmes aberrants du piano qui semble remplir une fonction d'accompagnement. 
Le mouvement VI renvoie au début de la composition : la flûte explore les séquences intervallaires mais avec une intention plus soutenue que précédemment comme on le voit dans les rythmes répétés des mesures 189-190 et 191-192. Puis c'est la répétition de toute une séquence rythmique de la mesure 236 à la fin du mouvement VI tandis que le mouvement s'accélère jusqu'au moment de rupture inattendu ; la rupture étant d'ailleurs le mode syntaxique privilégié chez Bonnefoy.

Dans le mouvement VII qui est rattaché au mouvement III, la flûte joue plus vite, dans un registre plus haut et plus urgent. À la fin du mouvement VIII, les relations mélodiques sont progressivement inaudibles, voire absentes, générant chez l'auditeur le sentiment d'être perdu. Tout au long de sa composition Jeremy Thurlow a su évoquer le mystère si cher à Bonnefoy. Dès le premier mouvement de "Plus avant que l'étoile ", la tonalité du morceau intrigue par son usage de sonorités inédites. Étant donné qu'il n'y a pas d'altération à la clé, le compositeur prend plaisir à moduler. L'effet est indubitablement contemporain. ${ }^{13}$ On note de grands écarts (deux octaves dans le quintolet) pour la flûte dans les notes de la mesure 8 qui correspond au syntagme "dans le reflet " : comme pour marquer, au plan compositionnel, la distance entre ce reflet et la réalité, distance qui est soumise à un traitement dramatique dans ce poème. En effet, le poème " Deux couleurs $»^{14}$ met en scène une problématique chère à Bonnefoy : celle de la représentation ou de l'image d'une part et de la Présence d'autre part, qui sont concomitantes. Résumant le mystère de l'origine et de la fin, un désir inassouvi, qui n'en finit pas d'être désir, la Présence est une expérience vécue comme l'Événement ultime de sa quête poétique, son horizon : horizon lointain que laisse entendre la musique par la magie de laquelle la présence est incontestablement présence, c'est-à-dire ambiguë, "s'échapp[ant] toujours plus loin, toujours au-delà ", dans ce lointain qui est "l'exposant commun qui frappe plus ou moins toute musique" (Jankélévitch 1983a, 64).

Dans « Deux couleurs ", l'irréel (ou le rêve) et l'inaccessible (ou l'éternité) que représente l'étoile s'offrent à travers le reflet de celle-ci. " La présence immobile de l'étoile " n'est qu' "un reflet d'étoile » ou une image " en reflet ». Or en cherchant à saisir l'étoile qui se reflète dans l'eau et confronté à la nature purement illusoire, stérile du reflet, le locuteur se surprend à croire en la présence de ce qui n'est qu'un leurre, en la vie de ce qui n'est qu'un rêve. Le reflet, en tant qu'image du leurre de la présence, en tant qu'effet de la présence, elle-même effet de langage, en tant qu'occultation de la réalité par l'image donne lieu à une thématique ruminante et lancinante. D'ailleurs, dans le mot " couleurs » on ne peut s'empêcher d'entendre 〈 leurre ` : question de vigilance. Mais la proximité phonétique, qui imite la fallacieuse proximité du reflet, peut se prêter à toutes sortes de divagations puisque dans " couleurs " on entend aussi c coule l'heure .. Dans la quatrième strophe, les mondes "s'écoulent». En témoigne le verbe ‘ passer > de la deuxième strophe, baudelairien s'il en est, qui noue le principe vital et fatal, le paradoxe du mouvement de la vie et de la mort, de l'apparition-disparition. Et de < passer > à < passer pour > il n’y a qu'un pas : « le pas baudelairien de l'amour des choses mortelles ", dirait Bonnefoy (1980, 121).

La première strophe évoque la fascination pour le reflet, le désir que le reflet puisse passer pour la chose même. Cependant, la quête de la présence doit composer, si l'on peut dire, 
avec le leurre : ils sont inextricables; en effet, pas de présence sans cette doublure qu'est le leurre : un leurre qu'il importera pourtant de briser ${ }^{15}$ La musique s'en chargera, qui brise le sortilège de l'écriture en même temps qu'elle le joue et le déjoue (pour parler comme Barthes). Les verbes ‘ prendre > et > bouger > dans la troisième strophe anticipent l'énergie musicale. La forte qualité événementielle de ce qui se joue ici, dans le poème, se fait par le truchement $\mathrm{du}$ ( froissement ) dont les sonorités graves, feutrées, estompées et sombres gagnent en résonance vibratoire, révélation d'une présence qui pourtant se dérobe. Ce froissement qui laisse apparaître un seuil à la surface de "l'eau fermée " figure l'instant paradoxal de la présence ou la présence paradoxale de l'instant, qui est finalement le paradoxe de la présence : instant intense, cependant euphémique (et euphonique) en même temps, celui d'une espérance qui sommeille. Dans cette même strophe, troubler l'eau revient à réactiver et vivifier le système langagier, à dissiper le mensonge, à < éveiller > le monde. ${ }^{16}$ Le leurre consiste à penser que l'image poétique est un seuil. Le geste de la main se voit doté d'une forte valeur performative : c'est la parole poétique telle que la conçoit Bonnefoy, parole qui, rompant le charme de l'image, fait advenir la présence. Le paradigme poético-performatif est bien celui de l'altération. Ici s'énonce la préoccupation bonnefoyenne par excellence : la poésie, loin d'être un repli du dire dans ses codes ou un éloignement du réel, doit nous y reconduire, renouer avec ce dont nous prive le langage, d'obédience conceptuelle. Ce qui explique le geste interventionniste du poète-locuteur ici, comme s'il faisait irruption dans sa création pour en briser les prestiges et retisser une autre proximité de la réalité.

Le phénomène acoustique, en ses combinaisons sonores difficilement jouables, redoublera, prolongera le geste poétique. Cette main qui met en branle la signifiance est aussi celle du musicien, pianiste et flûtiste, qui se doit d'être agile dans cette composition musicale. En effet, celle-ci exige de lui des prodiges d'adresse, des doigtés invraisemblables, une kinesthésie virtuose. Les mains et doigts s'imposent comme synecdoques d'un agir performatif qui scelle l'alliance de l'incarnation et de l'incantation dans le dépassement de l'image.

On pourrait aller jusqu'à dire que le reflet est en quelque sorte rédimé par la musique qui le plonge dans la réalité matérielle (et) mortelle, qui en perce l'illusion. "Ce pays » marque l'enracinement dans une topique spatio-temporelle, forcément contingente : un ici-maintenant où se joue le drame, l'affrontement de la vie et de la mort que la musique saura restituer, elle qui nous plonge dans l'expérience et la présence; nous y sommes ensemble dans l'audition de la musique. La main musicienne actualise le froissement engendré par le poète-locuteur, fait bouger cette présence, ouvre ce seuil, faisant jouer la dialectique essentielle : apparition/disparition, plénitude/manque, singularité/universalité, présence/ absence, désaisissement/ressaisissement, vie/mort, qui relèvent du vaste paradigme de l'inachevable.

Bien qu'il ne soit nullement question d'exploiter les ressources de l'harmonie imitative, les changements de mesures intriguent (souvent des mesures quasi vides ou en fin de mouvement), qui favorisent ainsi l'éclosion d'un mystère. Il n'est pas fortuit non plus que le cri de la dernière strophe soit porté par le tempo sempre con moto avant de finir sur le déploiement de l'aube molto largo sous l'égide de la flûte. 
Grand vocable de la poétique de Bonnefoy, grand principe salvateur et salutaire de l'image, l'incarnation, prendra sa pleine valeur dans les chants composés par Thurlow deux ans plus tard, qui ouvrent les poèmes à la visibilité mais une visibilité qui comporte, phénoménologiquement parlant, une doublure d'invisibilité. Inspirée du recueil Les planches courbes (2001), l'œuvre s'intitule Quiet Songs (pour soprane, clarinette, contrebasse et harpe), qui comprend "La pierre " (tiré du poème " Je déplace du pied ", Bonnefoy 2001), "Effacement " (tiré de "Un même effacement ", Bonnefoy 2001) et "Pluie " (tiré de "Pluie des matins d'été ", Bonnefoy 2001). ${ }^{17}$ Thurlow fut saisi par l'impression de présence, si tangible, qui se dégageait des poèmes. Là encore, il s'attache à restituer l'essence de l'œuvre poétique par le recours à des techniques qui semblent relever d'un régime imitatif. On a l'impression que les paroles guident la composition dans le but de donner toute son ampleur à la poétique de la voix bonnefoyenne. Dans le premier mouvement, "La pierre " 18 , l'homophonie du début cède le pas à la confusion, au hasard ; il n'y a aucun instrument pour ancrer et harmoniser la totalité. ${ }^{19}$ Une telle confusion dans la texture semble illustrer le verbe "troubler " (dans la troisième strophe), qui renvoie au brouillage d'une perception autre, affranchie des codes et cadres ordinaires. On note une ressemblance analogique entre le rythme musical et le rythme de l'action : ainsi, de grands intervalles imitent l'acte de déplacer du pied. Des irrégularités dans le rythme de la harpe font écho au terrain caillouteux et cahoteux. Un plus grand intervalle après "Cette large " (il s'agit d'une pierre) confere au mot un pouvoir de résonance. La certitude du 'c'est vrai' dans la deuxième strophe est portée par un sforzando. Le "soudain " qui clôt cette même strophe fait presque l'effet d'un saut en hauteur sonore. Il semble que musicalement il se produise, après un registre plus bas, l'épiphanie dans la hauteur à laquelle les lignes vocale et instrumentales jouent le «beau » du "Comme il fait beau ». Une harmonie diatonique, diffuse, s'installera.

«Effacement " (que Thurlow a composé en premier) fait entendre une angoisse atténuée, amortie. L'effet de trémolo génère une confusion qui est en quelque sorte ‘ effacée > par la nature soutenue de la partie vocale. Les silences imprègnent ce mouvement qui nous plonge dans un régime de pensivité conformément à la tonalité douce (quiet, attentive) que lui a conférée le compositeur. Le recours au diminuendo actualise l'évaporation de la deuxième strophe. L'، ailleurs , où se meuvent les colombes bénéficie du pincement des cordes de la harpe avec les ongles, ce qui produit un son plus éthéré, voire magique. On a l'impression d'une hausse de la tonalité qui restitue la syntaxe de Bonnefoy, syntaxe disloquée, heurtée, seule capable de laisser émerger la présence. La composition semble reproduire l'effacement, la dépersonnalisation du sujet de l'énonciation, insituable, qui est une voix plus qu'un sujet à proprement parler, une sorte de vacillement entre ، être et ne pas être , ; ainsi que d'autres miracles corrélationnels.

Avec "La pluie ", on entend, par une sorte d'harmonie imitative, le tambourinage, le tapotement. Bien vite, le mot « été » se voit propulsé, qui laisse entrevoir l'espoir. Parvenu au " rêve ", dans le troisième vers, la musique se fait onirique. Cependant, la matérialité ne s'y trouve pas niée puisque le mot " chaleur " s'installe à la mesure 31 dans le chaud registre du soprano, puis de la clarinette. Dans le dernier vers, le verbe "se répandre " résonne dans le 
murmure de sa dernière syllabe et l'échelonnement des instruments. Une composition qui fait la part belle à la Présence, en sa fulgurance.

En 2016, dans le sillage de ses compositions d'obédience bonnefoyenne, Thurlow créa "Over the Frost» (pour sextuor vocal, flûte alto et harpe), adapté de Collects for Lent de Stephen Romer, un cycle de poèmes lapidaires introspectifs qui dramatisent une crise spirituelle. Si l'entreprise de Thurlow réussit - comme en témoignent les réactions émues de Bonnefoy et Romer -, c'est parce que, pour lui, la mise en musique se conçoit comme un accompagnement, une émanation, voire un parallélisme. ${ }^{20}$ De son propre aveu, le défi fut de ré-imaginer musicalement le déploiement du poème, au fil des vers et des strophes. Pour Thurlow, la partition ne serait en somme que l'équivalent d'un scenario ou d'un plan d'architecte, " not the real thing ", prend-il soin de préciser : chez lui, comme chez Bonnefoy, il y a refus de l'enfermement dans la structure. L'expérience musicale consiste donc à s'en émanciper, qui noue la désécriture et la réécriture par l'intermède de la voix, métonymique du corps, cette voix qui lutte avec l'écriture.

La problématique de l'incarnation et de la présence chez Bonnefoy rejoint la vocation charnelle de la musique (qui n'abolit certes pas ses aspirations spirituelles), et la vocation charnelle de la parole. Raymond Court accorde à la musique un ‘ primat > dans sa tentative de penser la chair, qui favorise l'épanouissement du primat de la sonorité chez le poète : c'est bien là que surgit la Présence, par l'entremise de la vocalité et du rythme, certes, et de la dimension métonymique de chaque instrument, mais aussi dans «la phénoménalité spatio-temporelle de la musique " (Villela-Petit 1998). ${ }^{21}$

Dans la musique vocale ou instrumentale se dit le mystère de la temporalité, se creuse le paradoxe de cette lutte perdue mais menée néanmoins, se formulent des intuitions informulables. Peut-être Jeremy Thurlow s'est-il approché de ce que Bonnefoy nomme la ‘ vérité de parole , lui qui cherchait un dépassement, une invalidation des structures de la langue pour faire ressortir la présence, bafouée par la langue ; lui qui s'efforçait de relancer le poème sur la voie/voix de la poésie, ouvrant celle-ci à ce qui est hors d'elle, à un toujours plus réel qu'elle. L'expérience musicale accomplit le miracle d'un ravissement extatique : nous voici plongés dans l'insondable profondeur du réel, dans le mystère de l'instant et de la présence, ${ }^{22}$ où se joue constamment le drame de notre paradoxale et douloureuse condition ; l'on s'y éprouve vivante et mortelle, dans la complicité avec la vie, dans l'affrontement avec la mort. La musique restitue le mystère du monde et de notre condition, esquissé dans la poésie. Événement au sens plein du terme, elle facilite l'avènement à cette réalité plus intense, plus vraie, que Bonnefoy nomme la Présence. Prodige de la musique qui rompt en même temps qu'elle exerce le charme (au sens de ‘ carmen `) : elle nous ramène dans le temps de notre finitude en même temps qu'elle nous donne un aperçu de l'éternité ; elle nous restitue le rapport direct et intime à la présence, elle accomplit le miracle du signe devenant lieu et présence, elle scelle l'alliance du signe et du sensible.

La présence, expérience dont Bonnefoy réveille le désir chez son lecteur et Thurlow chez son auditeur, est précarité ; désir que la composition musicale présentifie, amplifie et densifie, propulsant la poésie au-delà du poème vers plus que le langage, vers la présence, qui n’en 
finit pas de se dérober, tout comme l'horizon. Que Michèle Finck fasse de "la précarité de la musique [...] la condition même de la révélation » (Michèle Finck 2014, 280) ne saurait nous surprendre.

La musique joue (et joue sur) les paradigmes phénoménologiques de l'inachevé, de l'inachèvement, de l'inachevable, de l'inépuisable et de la suspension. Ce n'est pas un hasard si Bonnefoy a intitulé ses entretiens sur la poésie, L'Inachevable ${ }^{23}$ puisque la poésie y est vouée. Le phénomène, quelles que soient ses modalités (musicale ou poétique), se gorgeant de tous les possibles, aura toujours une part d'indéfinissable, d'insaisissable, d'inachevable. La poésie, pour lui, jamais ne s'envisage seule. Bonnefoy entreprend de délinéer une communauté esthétique qui engloberait sans doute "Le Mystère de l'instant ${ }^{24}$ de Dutilleux ainsi que Jankélévitch et sa fascination pour Debussy et le mystère et bien sûr l'ineffable dont il faisait le mystère de la musique (cf. Jankélévitch/Berlowitz 1978) ${ }^{25}$. Pour le philosophe-musicologue, il y a bien un mystère de la sonorité : ce nouage de la Présence et du mystère, Jankélévitch l'a résumé sous le titre de Présence lointaine. Pour Bonnefoy, croire à l'instant, c'est croire à l'instant de la mort et à la mort de l'instant ; mystère qui n'en finira pas de nous hanter en sa poignance. Quant à la Présence, elle est « le paradoxe de la proximité lointaine, de la longinquité prochaine " (Jankélévitch 1983b, 63). Il nous incombe de recueillir ce mystère comme une offrande car c'est bien ainsi que Bonnefoy concevait la poésie. La composition musicale saura jouer le rôle d'intercesseur : " possibiliser l'impossibilité d'une coïncidentia oppositorum» (Jankélévitch 1983b, 53).

\section{Notes}

1 Carole Bourne-Taylor est Maître de conférences en littérature francophone à Brasenose College, Université d'Oxford. Elle tient à remercier vivement Benjamin Chaix-Rodier, Christian Wilson, Fleur Snow et Morgan Overton pour leur soutien musicologique.

2 Outre Bonnefoy, il convient d'ajouter d'autres inspirateurs, tels que Stephen Romer, poète, critique et traducteur de Bonnefoy ; Virginia Woolf ; John Keats ; Emily Dickinson ; John Burnside ; Luis Omar Salinas. Notons que Thurlow a composé deux œuvres inspirées de Bonnefoy. L'impulsion est souvent donnée par une découverte littéraire, telle que la lecture de Bonnefoy en 2012. Pour ce qui est de "Memory is the Seamstress " (string quartet), adapté d'Orlando de Virginia Woolf, il fut commandité par Emma Sutton qui dirige le projet Virginia Woolf \& Music à St Andrew's. Ainsi en fut-il pour "A London Street in Winter » (song), inspiré de "Street Haunting: A London Adventure ".

3 (Imaginaire , en tant que " réseau où le sens est dans la relation ». Tout y est processus et traces relationnels. (Cf. Thomas 1998, 15)

4 Larticle de Quillier présente un portrait de Bonnefoy en musicien ou maître de chapelle, compositeur, chef de chœur, chef d'orchestre. L'acousmatique s'attache à développer le sens de l'écoute ; les acousmates sont des sons inchoatifs. 
5 Ainsi peut-on lire dans une lettre de Virginia Woolf à la violoniste Elizabeth Trevelyan, à propos de la structure de son Roger Fry: A Biography: «I always think of my books as music before I write them." (Woolf 1980, 425-426)

6 «[... taken as a whole, the poem offered too much, and would overwhelm me " [interview téléphonique entre J.T. et C.B.-T., juin 2018] : le verbe et l'intensificateur signalent que l'on est dans le registre de l'émotion, voire dans la redondance émotionnelle. Au fil d'un cheminement compositionnel qui a consisté à explorer les images et énergies de chaque strophe, Thurlow a isolé la première partie du poème qui présente une forme dramatique, différente du poème dans son intégralité, ayant sa propre intégrité.

7 Thurlow nous offre des éléments paratextuels : le poème de Bonnefoy, nous dit-il, était « d'une beauté envoûtante » - l'expression " hauntingly beautiful » [interview téléphonique entre J.T. et C.B.-T., juin 2018] en anglais a l'avantage de cristalliser d'autres associations et connotations. Il ne s'agissait nullement de transcrire une quelconque émotion étant donné la complexité du phénomène de l'inspiration.

8 Thurlow voulait que les musiciens puissent « ressentir > les sons, la tonalité musicale (le fameux mood qui demeure intraduisible) que la musique s'attache à restituer.

9 Nous sommes ici dans le paradigme édicté par Michel Deguy dans son thrène À ce qui n'en finit pas. Cf. aussi Jenny $(1990,144)$ pour qui l'élégie se situe «dans l'imminence indéfiniment suspendue de la fin".

$10 \mathrm{La}$ discordance fut érigée en paradigme par Baudelaire, dans sa "Cloche fêlée " (Les Fleurs du mal).

11 «Et que fait-elle, alors, la poésie ? Elle tente de réveiller ces présences dormantes sous les concepts, ce qui nous rend présents à nous-mêmes, qui alors ne sommes plus dans l'espace de la matière mais dans un lieu, elle substitue ce lieu au dehors du monde, elle fait de ce dehors une terre. La poésie n'est pas un dire, mais un déblaiement, une instauration ». (Bonnefoy 2010b)

12 L'eau et le reflet sont casés sur un mi aux mesures 187 et 210 respectivement, tandis que l'étoile est sur la même note, un sol.

13 Un exemple en est la mesure 49 avec du mi bécarre au piano main droite, mais du mi bémol à la flûte et cela sur une même hauteur de portée : l'on obtient par ce procédé une seconde mineure qui perturbe l'oreille; ce qui se produit à plusieurs endroits dans la partition.

14 Ces couleurs, qui renvoient aux tableaux de Poussin, Moïse sauvé des eaux, fonctionnent au sein d'une dynamique contrapuntique, d'affrontement et de relativisation. De cette interaction jaillit une autre dimension. En adaptant ce poème, Thurlow lui aurait donc restitué sa pleine valeur, sa dimension cachée : on obtient un objet pluridimensionnel en quelque sorte, objet qui s'ouvre à son double destin de vie et mort. Il convient de noter que Dans le leurre du seuil contient une structure dialectique : au monde-image des mots s'oppose un espace ouvert.

15 En effet, cette conception de la poésie est " une conception dialectique, où dans un premier mouvement rêveur elle se donnerait à l'image, mais pour critiquer celle-ci ensuite, au nom de l'incarnation, pour la simplifier, pour l'universaliser, pour finir par l'identifier aux données simples de l'existence» (Bonnefoy 1990, 17). 
16 Cf. Bonnefoy (1990, 191-192) : «L'Image est certainement le mensonge, aussi sincère soit l'imagier".

17 Thurlow avoue que ces chants, composés en 2014, résultent d'un tâtonnement. Les deux premiers poèmes ("Je déplace du pied » et «Un même effacement ») sont tirés de la section " La Pluie d'été » le troisième poème ("Pluie des matins d'été ») de la section «La pluie sur le ravin ».

18 La pierre est toujours le signe d'une affinité avec le réel, avec le lieu dans l'univers imaginaire de Bonnefoy.

19 Aucun centre gravitationnel dans l'harmonie. La contrebasse joue au-dessus des autres.

20 Cf. Paul Dukas : "On ne met pas les poèmes en musique. On donne un accompagnement aux paroles, et c'est bien autre chose. La première idée, en effet, suppose une fusion; la seconde constate un parallélisme ». (cité dans Gribenski 2004 ; de la Revue Musica, mars 1911, réponse à l'enquête de Fernand Divoire: "Sous la musique, que faut-il mettre ? [...] ")

21 Raymond Court parle de "la coalescence profonde entre chair et musique " (Piettre 2003, 159). À cet égard, il convient de mentionner Court (1996) et Villela-Petit (1998). La phénoménologie fait grand cas de ce qui n'en finit pas d'apparaître sur un mode inchoatif.

22 Bonnefoy reprochait à Valéry de sacrifier " le mystère de la présence " dans un " art de la forme close ", (Bonnefoy 1980b, 103).

23 Selon Bonnefoy, la poésie transgresse le discours conceptuel et sa menace d'aliénation en invitant à «faire apparaître dans les mots la présence des choses» (Bonnefoy 2010a, 342).

24 Poème instrumental pour orchestre à cordes, cymbalum et percussion (1989).

25 Jankélévitch emprunte l'expression à Rilke. Rappelons aussi cette citation « la musique ne signifie rien, donc elle signifie tout " (Jankélévitch 1983a, 19).

\section{Bibliographie}

Bonnefoy, Yves : "L'acte et le lieu de la poésie ". In : Bonnefoy, Yves : L'Improbable et autres essais. Paris : Mercure de France, 1980, 107-133. [=Bonnefoy 1980a]

Bonnefoy, Yves : «Paul Valéry ». In : Bonnefoy, Yves : L'Improbable et autres essais. Paris : Mercure de France, 1980, 99-105. [=Bonnefoy 1980b]

Bonnefoy, Yves : «Deux Couleurs» (1975). In : Bonnefoy, Yves : Poèmes. Paris : Gallimard, 1982, 271-274.

Bonnefoy, Yves : La vérité de parole. Paris : Mercure de France, 1988.

Bonnefoy, Yves : Entretiens sur la poésie, 1972-1990. Paris : Mercure de France, 1990.

Bonnefoy, Yves : Le nuage rouge (1977). Paris : Mercure de France, 1992.

Bonnefoy, Yves : «Entretien avec Patrick Kéchichian ». In : Le Monde (7 juin 1994), 2.

Bonnefoy, Yves : «Mallarmé et le musicien». In : Finck, Michèle (éd.) : Yves Bonnefoy. Poésie, peinture, musique. Strasbourg : PU de Strasbourg, 1995, 7-21.

Bonnefoy, Yves : La pluie d'été, Les Planches courbes. Paris : Gallimard, 2001. 
Bonnefoy, Yves : "Poésie, peinture, musique" (1996). In : Gagnebin, Murielle (éd.) : Yves Bonnefoy. Lumière et nuit des images, suivi de "Ut pictura poesis" et autres remarques par $Y$. Bonnefoy. Seyssel : Champ Vallon, 2005, 313-340.

Bonnefoy, Yves : Linachevable. Entretiens sur la poésie 1990-2010. Paris : Albin Michel, 2010. [=Bonnefoy 2010a]

Bonnefoy, Yves : "L'inachèvement est ce qui caractérise la poésie. Entretien avec Philippe Delaroche et Baptiste Liger ». In : L’Express (22 novembre 2010), https:/www.lexpress.fr/culture/ livre/yves-bonnefoy-l-inachevement-est-ce-qui-caracterise-la-poesie_937936.html (consultation 25.02.2019). [=Bonnefoy 2010b]

Court, Raymond : «Musique et chair ». In : Conférence 3 (30 octobre 1996), http://www.revue-conference.com/index.php?option=com_content\&view=article $\& i d=364:$ musique-et - chair $\&$ catid=35\&Itemid=165 (consultation 25.02.2019).

Court, Raymond : «Pour une esthétique musicale». In : Piettre, Bernard (éd.) : Kairos 21. Philosophie et musique. Toulouse : PU de Toulouse, 2003, 153-170.

Court, Raymond : "Musique, voix et chair». In : Poizat, Michel / Cusin, Michel / Baas, Bernard / Didier-Weill, Alain (éds) : Musique et sacré. Ambronay : Ambronay Éditions, 2005, 69-82.

Deguy, Michel : À ce qui n'en finit pas. Paris : Seuil, 1995.

Finck, Michèle : "Poétique de la voix rauque ». In : Finck, Michèle (éd.) : Yves Bonnefoy. Poésie, peinture, musique. Strasbourg : PU de Strasbourg, 1995, 33-42.

Finck, Michèle : Poésie moderne et musique. Essai de poétique du son. Paris : Champion, 2004.

Finck, Michèle : Épiphanies musicales en poésie moderne, de Rilke à Bonnefoy : le musicien panseur. Paris : Champion, 2014.

Finck, Michèle : "Scènes musico-théâtrales et ébauches de mystères ». In : Landi, Michela (éd.) : La double séance. La musique sur la scène théâtrale et littéraire. Firenze : University Press, 2017, 327-341.

Froidefond, Marik : «Bonnefoy et Dutilleux. Pour une nouvelle postérité théorique de Baudelaire ». In : Ergal, Yves-Michel / Finck, Michèle (éds) : Littérature comparée et correspondance des arts. Strasbourg : PU de Strasbourg, 2014, 117-133.

Gribenski, Michel : «Littérature et musique ». In : Labyrinthe 19,3 (2004), https://journals.openedition.org/labyrinthe/246 (consultation 25.02. 2019).

Jankélévitch, Vladimir : Debussy et le mystère de l'instant. Neuchâtel : Éditions de la Baconnière, 1950. Jankélévitch, Vladimir : La musique et l’ineffable (1961). Paris : Seuil, 1983. [= Jankélévitch 1983a]

Jankélévitch, Vladimir : La Présence lointaine. Albeniz, Séverac, Mompou. Paris : Seuil, 1983. [= Jankélévitch 1983b]

Jankélévitch, Vladimir / Berlowitz, Béatrice : Quelque part dans l'inachevé. Paris : Gallimard, 1978. Jenny, Laurent : La parole singulière. Paris : Belin, 1990.

Lassus, M.-Pierre : G. Bachelard musicien. Une philosophie des silences et des timbres. Villeneuve-d'Ascq : Presses universitaires du Septentrion, 2010.

Pinson, Jean-Claude : Habiter en poète. Essai sur la poésie contemporaine. Seyssel : Champ Vallon, 1995. 
Quillier, Patrick : "Entre bruit et silence : Yves Bonnefoy, Maître de Chapelle ? (esquisses acroamatiques)». In : Littérature 127 (2002) : L'oreille, la voix, 3-18.

Starobinsky, Jean : «Préface». In : Bonnefoy, Yves : Poèmes. Paris : Gallimard, 1982, 7-30.

Thomas, Joël (éd.) : Introduction aux méthodologies de l'imaginaire. Paris : Ellipses, 1998.

Villela-Petit, Maria : «La spatio-temporalité de la musique ». In : Chouvel, Jean-Marc / Solomos, Makis (éds) : L'espace. Musiquelphilosophie. Paris : L'Harmattan, 1998, 29-39.

Woolf, Virginia : The Letters of Virginia Woolf, VI, 1936-41. Éd. Nigel Nicholson et Joanne Trautmann. New York : Harcourt Brace Jovanovich, 1980.

\section{Discographie}

Thurlow, Jeremy: "Plus avant que l'étoile ", for flute and piano, after verses by Yves Bonnefoy. 17', 2012.

Thurlow, Jeremy: "Quiet Songs ", for soprano and instrumental trio (clarinet in Bb, double bass and harp). 6', 2014.

Thurlow, Jeremy: "Over the frost ", for six voices (S, MS, A, T, Ba, B) with alto flute and harp. 13', 2016.

\section{Sitographie}

https://jeremythurlow.com/worklist (consultation 25.12. 2020).

https://soundcloud.com/jeremythurlow (consultation 25.12. 2020). 